National Marine

Fisheries Service

NOAA
Fishery Bulletin

a established in 1881 o
Spencer F. Baird

First U.S. Commissioner of Fisheries and founder of Fishery Bulletin

\begin{abstract}
Mark-recapture studies using data collected at rotary screw traps (RSTs) are used to estimate abundances of migrating juvenile salmonids exiting natal rearing habitats. Frequently, environmental conditions and mechanical failures decrease RST efficiencies, or completely halt operations, leading to sparse and missing data. In this study, we show how a time-stratified hierarchical Bayesian model framework can incorporate prior information to increase the accuracy and precision of estimates made with sparse and missing data. To do this, we incorporated annually recurring salmonid emigration characteristics into the model using multiple years of data. We compared abundance estimates of the hierarchical multiyear model with 3 single-year Bayesian models, using simulated and real RST data. The hierarchical multiyear model

\author{
Bryce N. Oldemeyer (contact author) ${ }^{1}$ \\ Timothy Copeland ${ }^{2}$ \\ Brian P. Kennedy 3 \\ Email address for contact author: bryce@henrysfork.org \\ ${ }^{1}$ Henry's Fork Foundation \\ 801 Main Street \\ Ashton, Idaho 83420 \\ 2 Idaho Department of Fish and Game \\ 600 South Walnut Street \\ Boise, Idaho 83686 \\ 3 Department of Fish and Wildlife Resources \\ University of Idaho \\ 875 Perimeter Drive \\ Moscow, Idaho 83844
} was as accurate and precise as the best model when data were complete and abundant, but outperformed other models when data were sparse and missing for multiweek blocks. For species with low abundances or low detection efficiencies, the hierarchical multiyear model used data from all years and recurring emigration characteristics to increase the accuracy and precision of estimates. This model is a valuable tool for fish and wildlife biologists who repeat mark-recapture studies annually and encounter sparse and missing data.

Manuscript submitted 5 January 2018. Manuscript accepted: 10 May 2018.

Fish. Bull. 116:254-265 (2018)

Online publication date: 7 June 2018. doi: 10.7755/FB.116.3-4.4

The views and opinions expressed or implied in this article are those of the author (or authors) and do not necessarily reflect the position of the National Marine Fisheries Service, NOAA.
To effectively manage free-ranging animals, information on survival rate, population growth rate, and recruitment are needed to understand factors influencing populations (Fryxell et al., 2014). It is often necessary to know abundances during various life stages to calculate this information but obtaining censuses of natural populations is difficult (Seber, 2002). Studies structured around sighting, capturing, or counting individuals and expanding these counts based on detection or implemented to estimate abundances when a census is not feasible (Nichols, 1992; Mill, 2007). These types of mark-recapture studies have broad application and have been used to estimate abundances of blue whales (Balaenoptera musculus) and humpback whales (Megaptera novaeangliae) (Calambokidis and Barlow, 2004), grizzly bears (Ursus arctos) (Mowat and Strobeck, 2000), herbivorous insects (Kareiva, 1983), and numerous other species so that marked indisampling efficiencies are regularly

\title{
A multiyear hierarchical Bayesian mark-recapture model incorporating data on recurring salmonid behavior to account for sparse or missing data
}

viduals in the population can be detected during later sampling periods.

The Lincoln-Petersen model is foundational for estimating abundances using mark-recapture data where unmarked abundance, $U$, is estimated using the number of marked individuals in the population, $n$, the number of unmarked individuals counted or captured at a sampling event, $u$, and the number of marked individuals counted or captured at a sampling event, $m$ :

$$
U=n \cdot u \cdot m^{-1} .
$$

Lincoln-Petersen model assumptions can be difficult to satisfy, primarily the assumption of equal capture probability throughout a sampling period, necessitating modifications to the model. Commonly, mark-recapture studies occur continuously over time where individuals are marked, captured, and recaptured for several weeks or months. The accuracy of an abundance estimate obtained from pooling data relies on the assumption that capture prob- 
abilities are homogenous throughout the sampling period. Changes in the environment, species behavior, or sampling equipment can alter capture efficiencies, violating the assumption of homogenous capture probabilities and lead to biased abundance estimates. Sampling periods are often stratified temporally into smaller, more homogenous stratum for computation and later summed to minimize the violation of the capture probability assumption (Otis et al., 1978; Schwarz and Taylor, 1998). This stratification approach can be effective when sufficient data are available, but stratifying sparse data can lead to wide confidence intervals or estimation failure resulting from division by zero when individuals are not recaptured during a stratum (Seber, 2002).

When mark-recapture data are sparse or missing, hierarchical Bayesian models have proven to be an effective approach for obtaining abundance estimates (Mackey et al., 2008; Royle et al., 2011; Sethi and Tanner, 2013). The Bayesian paradigm provides a framework to incorporate prior biological knowledge into models by using hierarchal structures between parameters and by specifying informative prior distributions (Ellison, 2004). When prior biological information is available, structuring models to incorporate this information can produce more accurate and precise estimates (Royle and Dorazio, 2008). Mäntyniemi and Romakkaniemi (2002) implemented a hierarchical Bayesian model to estimate Atlantic salmon (Salmo salar) smolt abundances in the Conne River, Canada, and River Tornionjoki, in northern Scandinavia, while accounting for overdispersion associated with the species schooling behavior. Bonner and Schwarz (2011) increased the precision and accuracy of abundance estimates of Conne River Atlantic salmon smolts by parameterizing the expected abundances of smolts as a smooth function of time, using penalized Bayesian splines (P-splines) to address sparse data. These single-year models are effective with sparse data and short periods of missing data but fail to use or incorporate all the information available in long term monitoring data sets.

The goal of our study was to illustrate how a timestratified hierarchical Bayesian model framework can incorporate prior years of information to increase the accuracy and precision of estimates made using sparse and missing data. To achieve this goal, we took a 2-step approach. First, we compared the performance of 3 Bayesian models that had within-year structures and 1 Bayesian model with a between-year hierarchical structure by using a simplified data set exhibiting various degrees of sparse and missing information roughly similar to real data. By using a simplified data set constructed from known parameters, we were able to quantify model performance by comparing the approximate posterior distributions produced by each model to the known parameters used to create the simplified data set. To illustrate how the concept works with real data, we then compare abundance estimates of the 4 models to 2 data sets, one set with complete and abundant data and another set with sparse and missing data. Covariates that were suspected to influence migration characteristics and juvenile abundances between years, e.g., seasonal hydrographic fluctuations and previous year counts of redds (nests dug by salmon in river beds), were excluded from both the data sets and model formulas in order to illustrate the basic concept and functionality of the competing models. After we compared the use and functionality of competing models, we discuss the various ways covariates could be included and how models could be extended to address more specific scenarios.

The model that used multiple years of data via the between-year hierarchical structure was able to bridge large periods of missing data (upwards of several weeks in some years) and sparse data by using the annually recurring emigration characteristics expressed by the species in the study, juvenile Chinook salmon (Oncorhynchus tshawytscha) in Idaho, to produce the most accurate and precise estimates. To our knowledge, this is the first time multiple years of data have been used to increase the robustness of abundance estimates calculated from sparse and missing mark-recapture data based on annually recurring behavioral characteristics.

\section{Materials and methods}

There are 2 primary components to our study. First, we compare estimates produced from 3 single-year Bayesian models and 1 hierarchical multiyear Bayesian model, using simulated scenarios reflective of missing and sparse data typical for monitoring with rotary screw traps (RSTs) in Idaho. To illustrate a proof of concept on how the structures of the Bayesian models function, models where simple (e.g., did not include environmental covariates or individual movement parameters) and data for simulated scenarios were not stochastic. Parameter estimates produced for the simplistic simulated data scenarios were compared with the known parameters used to create the simulated data to evaluate bias and precision for each of the models. Next, we demonstrate how models performed with real juvenile Chinook salmon data collected at Marsh Creek and Big Creek, Idaho, which reflect good and poor quality data sets. We used the full data record from the initial year of trap operation to 2014 to inform the estimates for the 2014 emigration.

\section{Field sampling and data collections}

Mark-recapture studies have been widely implemented to calculate anadromous juvenile salmonid abundances at RSTs (Zabel et al., 2005; Venditti et al. ${ }^{1}$; Copeland

\footnotetext{
${ }^{1}$ Venditti, D. A., J. Flinders, R. Kinzer, C. Bretz, M. Corsi, B. Barnett, K. A. Apperson, and A. Teton. 2012. Idaho supplementation studies: brood year 2009 synthesis report, August 1, 2009-July 31, 2011. Idaho Dep. Fish Game Rep. $12-13,24$ p. [Available from website.]
} 
et al. $^{2}$ ). Since 1990 , over 150 RSTs have been deployed in the Columbia River basin. Many RSTs operating for more than 20 years (Pacific States Marine Fisheries Commission, Columbia Basin PIT Tag Information System, database available from website, accessed July 2015). Abundance estimates obtained from RST data are used to assess population productivity from the freshwater habitat and as the foundation for other life-cycle performance metrics (e.g., Venditti et al.1; Copeland et al., 2014). This demographic information is central for monitoring salmonid populations and is often used to inform conservation actions (Venditti et al. ${ }^{1}$; Copeland et al. ${ }^{2}$ ).

Rotary screw traps are passively operating traps constructed of a partially submerged cone mounted to 2 pontoons (Johnson et al., 2007). The pontoons enable a RST to float while the cone funnels fish into a holding box located at the stern of the RST. Once fish are in the holding box, a helical twist within the cone prevents individuals from swimming upstream and out of the trap. When a RST is in operation, a trap tender removes captured fish from the holding box. Targeted individuals are anesthetized, inspected for tags, and length and weight data are recorded (Johnson et al., 2007). An allotted number of individuals are marked and released upstream of the RST (for a single trap design) or downstream (for a double-trap design) to be recaptured during proceeding days.

Rotary screw traps in Idaho typically operate from early March until freezing temperatures and frazil ice make them inoperable in November. The exact dates of RST installation and removal are dependent on yearly environmental conditions and sampling duration can vary by 1 or 2 weeks among years. Unexpected ice, high water, and RST mechanical failures can reduce trap efficiency or halt RST operations for several days to several weeks within a year. During these outages, it is assumed fish continue to pass the RST because this migratory behavior was observed during previous years. Most stream-type Chinook salmon in Idaho follow a recurring bimodal migratory pattern where the majority of a cohort begins emigration out of headwater rearing habitat in the fall and the remainder of the cohort emigrates in the spring (Bjornn, 1971, 1978).

\section{Parameterization of time-stratified mark-recapture models}

Data collection at RSTs consists of the daily number of unmarked and marked fish captured, and the number of marked fish released the day prior. Schwarz and Bonner ${ }^{3}$ found that weekly stratification of RST

\footnotetext{
${ }^{2}$ Copeland, T., R. V. Roberts, B. N. Oldemeyer, and K. A. Apperson. 2013. Idaho steelhead monitoring and evaluation studies: annual progress report, January 1, 2012-December 31, 2012. Idaho Dep. Fish Game Rep. 13-07, 47 p. [Available from website.]

${ }^{3}$ Schwarz, C. J., and S. J. Bonner. 2012. An application of a Bayesian stratified-Petersen model to estimate the number of outgoing fish on the Cheakamus River, British Columbia. Simon Fraser Univ. Rep. 2012-02-22 . [Available from website.]
}

mark-recapture data provided a sufficient balance between maintaining run characteristics while avoiding unnecessary data sparsity issues under the assumption that daily capture probabilities were similar within the week. Therefore, we opted to stratify year $(j=1, \ldots, t)$ by ordinal week $(i=1, \ldots, s)$. If capture probabilities were subject to high variability within a weekly stratum, stratum size could be decreased. For our model that used multiple years of data, the weekly stratification of the number of unmarked fish captured in the $i$ th stratum in the $j$ th year was denoted as $u_{\mathrm{ij}}$, the number of marked fish released in the $i$ th stratum in the $j$ th year as $n_{\mathrm{ij}}$, and the number of recaptured fish captured in the $i$ th stratum in the $j$ th year as $m_{\mathrm{ij}}$. For the 3 models that used data from 1 year, symbol designation remained the same but the subscript denoting year, $j$, was removed.

The likelihood function of time-stratified mark-recapture models implemented in the Bayesian framework consisted of 2 primary components: the probability an individual was captured at the RST and the estimated number of unmarked individuals passing the RST. The numbers of individuals recaptured in a stratum, $m_{\mathrm{ij}}$, were assumed to be binomially distributed by the number of marked individuals released upstream of the RST within the stratum, $n_{\mathrm{ij}}$, and the probability that an individual passing the RST was captured, $p_{\mathrm{ij}}$ :

$$
m_{\mathrm{ij}} \sim \operatorname{Binomial}\left(n_{\mathrm{ij}}, p_{\mathrm{ij}}\right) .
$$

Previous studies have modeled $m_{\mathrm{ij}}$ as a multinomial distribution incorporating an additional parameter describing the process of an individual's probability of being available for recapture during a later stratum (Mäntyniemi and Romakkaniemi, 2002; Bonner and Schwarz, 2011). We chose to exclude this parameter to simplify our models because $>96 \%$ of juvenile Chinook salmon recaptured at RSTs in our study did so within the proceeding day of release. The likelihood of the model is complete when the number of unmarked individuals captured within a stratum, $u_{\mathrm{ij}}$, is incorporated by using the binomial distribution:

$$
u_{\mathrm{ij}} \sim \operatorname{Binomial}\left(U_{\mathrm{ij}}, p_{\mathrm{ij}}\right),
$$

where $U_{\mathrm{ij}}=$ the estimated number of unmarked fish passing the RST during the stratum.

The assumptions of the time-stratified Lincoln-Petersen model are (Otis et al., 1978) as follows:

1 Individuals do not emigrate or die between marking and recapture;

2 Marks or tags are not shed;

3 Marks or tags are detected if present at recapture;

4 Marked and unmarked individuals within a stratum have the same probability of capture;

5 Individual movements within a stratum are independent; and

6 Individuals passing or being released below the RST are emigrating downstream and remain below the RST. 


\section{Model set}

We compared 4 models by using the constructed likelihood components outlined above with different parameter structures. The most basic model, $\mathrm{M}_{\mathrm{PS}}$ (pooled capture probability and simple abundance parameters), analyzed 1 year of data with a simple prior for $U$ where $\log \left(U_{\mathrm{i}}\right)$ shared identical normal priors with fixed mean and variance and where capture probability $(p)$ was pooled, $p_{\mathrm{i}}=p$ for $i=1, \ldots, s$ across all strata. The $\mathrm{M}_{\mathrm{PS}}$ model structures are functionally similar to a pooled Lincoln-Petersen model in that $p$ are structured to be pooled and constant across strata within the year and $U$ is independent between strata.

The second model was similar to models used by Mäntyniemi and Romakkaniemi (2002), which allowed information about $U$ and $p$ to be shared among strata within the year, and is denoted $\mathrm{M}_{\mathrm{HW}}$ (hierarchical within-year parameter structure). This model contained a hierarchical structure that assigned both $U$ and $p, \log \left(U_{\mathrm{i}}\right)$ and $\operatorname{logit}\left(p_{\mathrm{i}}\right)$, normal priors with common mean and variance within the year. The $\mathrm{M}_{\mathrm{HW}}$ hierarchical structures allow information about $U$ and $p$ to be shared across all strata within a single year.

The third model is structured from Bonner and Schwarz (2011) and uses the P-spline hierarchical prior for $U$ to smooth estimates between adjacent strata, localizing the sharing of information between adjacent strata within the year, and is denoted $\mathrm{M}_{\text {SPLINE }}$. A hierarchical structure for $p$ assigned $\operatorname{logit}\left(p_{\mathrm{i}}\right)$ normal priors, with common but unknown mean and variance within the year, was used for the $\mathbf{M}_{\text {SPLINE }}$ model. The $\mathbf{M}_{\text {SPLINE }}$ hierarchical structures allows information about $p$ to be shared among strata throughout the year but uses the temporal ordering of strata within the year to give greater weight to adjacent strata for sharing information in regards to $U$. Following the recommendations of Lang and Brezger (2004) and Schwarz and Bonner, ${ }^{3}$ we placed knot points evenly across strata at 4 -week intervals. These authors found that this spacing was a suitable compromise between spline overfitting and sharing information among strata. We chose to exclude the additional parameter used to model the probability that an individual is available for recapture in proceeding strata as explained above.

The hierarchical multiyear (between-year) model, $\mathrm{M}_{\mathrm{HB}}$, allows information from the same temporal period among years to be shared in regard to $p$ and $U . \mathrm{M}_{\mathrm{HB}}$ contains a hierarchical structure for $U$ and $p$, where $\log \left(U_{\mathrm{ij}}\right)$ and $\operatorname{logit}\left(p_{\mathrm{ij}}\right)$ have a common mean within strata of the same ordinal time period between years. By structuring the hierarchy between years, recurring run characteristics specific to each stratum (ordinal week for this study) were integrated into the model.

Selection of prior distributions for the highest level of the model hierarchies was chosen to be vague and weakly informative in regard to their parameters and to be identical to prior distributions used in previous research when applicable. Prior distributions selected also aligned with expert knowledge of and experience with
RSTs. For instance, capture probabilities at RSTs rarely exceed 0.5 and often average 0.1-0.2 depending on the trap location and time of year. The prior distributions selected for the hyperparameters of each capture probability for each model had a median of roughly 0.1 and $95^{\text {th }}$ percentiles from 0.0 to 0.5 . Additionally, prior distributions for the hyperparameters of each abundance parameter have a median abundance of roughly 22,000 with $95^{\text {th }}$ percentiles from 814 to 583,381 , with the exception of the $\mathbf{M}_{\text {SPLINE }}$ model that uses hyperparameters implemented by Bonner and Schwarz (2011). Complete structures for all models can be found in Supplementary Table 1 and Supplementary Figures 1-4.

\section{Test data sets}

A simplified data set roughly similar to RST data found in Idaho was created with known $U$ and $p$ parameters. The simulated data set used for the scenarios spanned 10 years $(j=10)$ with 35 strata per year $(i=35)$. This scheme roughly corresponds to the early spring transition in mid-March and the late fall transition in midNovember. The migration of juvenile Chinook salmon in Idaho typically has 2 pulses, one in the spring and one in the fall; therefore, parameters $U_{\mathrm{ij}}$ followed a smooth bimodal run with a small peak at strata 6 (mid-May) and the majority of the individuals centered on a peak at stratum 29 (late September). Parameters $p_{\mathrm{ij}}$ were constant at 0.333 for strata $1-4$ and 13-35, and constant at 0.111 for strata 5-12. As discharge increases in the spring, RSTs are often relocated out of the thalweg to slower portions of the river transect to avoid woody debris and decrease mechanical stress on the sampling equipment. In addition, as discharge increases, the relative amount of water sampled by RSTs decreases. These conditions often decrease trap efficiencies and are the justification for the decreased capture efficiencies for strata 5-12 in our simulated data. The number of marked individuals, $n_{\mathrm{ij}}$, released in each stratum was equivalent to the number of unmarked individuals captured, $u_{\mathrm{ij}}$, up to 50 individuals. The restriction to 50 individual was implemented to mimic tag and handling permit constraints that are common when dealing with threatened or fragile species at RSTs. Total yearly abundance summed over all 35 stratum was 23,477 .

This data set was modified to simulate realistic occurrence of sparse and missing data. The scenario using data produced from the parameters listed above was denoted as "full" owing to the completeness across strata and years and was the initial scenario in our simulation study used for subsequent modification. The second scenario is identical to the full scenario with the exception of information from strata 5-8 in the first year being removed. This 4 -stratum exclusion mimicked RST conditions when spring flows halt RST operations for several weeks. The third scenario had information from strata 5-8 removed from the first year in addition to reducing the number of marked and recaptured individuals by $60 \%$ across all strata 
and years. The $60 \%$ reduction typifies data for several RSTs that have low numbers of marked and recaptured fish because of site limitations or low abundances. The fourth scenario had information from 8 strata, strata $12-19$, removed from the first year of the data set and had a $60 \%$ reduction across all marked and recaptured individuals across all strata and years. This modification emulates several RSTs that did not operate during summer months initially because it was thought that salmonid migration had ceased during this time period (see history of the Marsh Creek data set below). It was later realized that small, but significant, numbers of individuals did migrate throughout the summer and RSTs now operate during this time period. Simulated scenarios and known parameters used to create the data are presented in Supplementary Table 2 and Supplementary Figures 5-8. Each model was tested with these 4 scenarios.

We illustrate the relative performance of the 4 competing models, using 2 RST data sets. Marsh Creek and Big Creek are tributaries to the Middle Fork of the Salmon River located in central Idaho, have comparable salmon populations, but have "good" and "poor" quality data sets, respectively.

Marsh Creek is a third-order tributary with a RST located at a river transect that has geographic and hydrographic features conducive to continuous operation throughout the majority of the migratory season with high capture efficiencies (Venditti et al. ${ }^{1}$ ). In addition, the RST has been operating for 21 years. Median day of installation is March 20 and removal is November 3 . Within this period, the RST operates a median of 97\% of the days. Gaps in operations are typically short for reasons such as icing and passing thunderstorms. During the first few years of operations, the RST was removed during the summer for 2.5 months until it was discovered that fish emigrated in that time period, too. This gap in the record motivated the fourth scenario in the simulations described above. Annual total catch of Chinook salmon has fluctuated more than 2 orders of magnitude from 846 to 91,719 fish. The high capture efficiencies, abundant amount of juvenile salmonids captured, and longevity of RST operation present a nearly ideal RST mark-recapture data set (Suppl. Fig. 9).

Big Creek, in comparison, is a fourth-order tributary located in the Frank Church River of No Return Wilderness. The RST was first installed in 2007 in a reach with widths from 30 to $40 \mathrm{~m}$, resulting in low capture efficiencies (Copeland et al. ${ }^{2}$ ). Trap efficiencies are much lower than those seen in Marsh Creek and can be quite variable (Suppl. Fig. 10). Annual total catch of Chinook salmon has fluctuated from 5167 to 33,308 fish. Median day of installation is March 11 and removal is November 10 . Within this period, the RST operates a median of $80 \%$ of the days. There are frequent short gaps in operations for reasons such as icing and thunderstorms that produce concomitant turbidity and debris. In addition, there are substantial gaps in operations during snowmelt. For example, adding up gaps in service $>7$ days, the RST missed a median of 55 days in mid to late spring, ranging from 20 to 75 days. The exception was 2007, the first year of operation, but the RST was not installed until May 21. The Big Creek RST data exemplifies nearly all potential pitfalls possible at RSTs in terms of sparse and missing data. Scenarios like those at the Big Creek RST are what motivated this study.

We focused on the results for the 2014 emigration year. During 2014, the Marsh Creek RST was deployed on March 22, 1 week later than the earliest date the trap had been deployed in its 21 years of operation. In 2014, the Marsh Creek RST ran continuously with high capture and recapture efficiencies throughout the trapping season and was removed on October 31 when snow and ice prevented operation. In total during 2014, the Marsh Creek RST missed 3 weeks of the trapping season at the tail ends when few fish were suspected to be migrating. In 2014, the Big Creek RST was deployed on March 13 and operated for roughly 5 weeks before high water prevented the RST from operating from April 11 to June 13. On June 14, the trap was redeployed and ran continuously until snow and ice prevented operation on November 9. During the 8-week outage, it was known that age-1 Chinook salmon overwintering in upper portions of Big Creek migrated out of the system during this time because this behavior had been observed in past years and at other RSTs in Idaho. In addition, when the Big Creek RST was operating, RST capture and recapture efficiencies where low owing to the site limitations described above. The Marsh Creek and Big Creek RST operations in 2014 exemplify the 1) complete and abundant mark-recapture data and 2) sparse and missing mark-recapture data.

\section{Model implementation}

All models were implemented with the statistical program JAGS (vers. 4.0.0; Plummer, 2003) run through the program $\mathrm{R}$ interface, vers. 3.2.2 ( $\mathrm{R}$ Core Team, 2015) with the R2jags package vers. $0.5-7$ ( $\mathrm{Su}$ and Yajima, 2015). The complexity of the models inhibited calculating an exact posterior distribution. As such, Markov chain Monte Carlo (MCMC) simulations were implemented in JAGS to sample from the joint posterior distributions of all parameters to approximate a posterior distribution. Three parallel chains initiated at random values were run for each model. Chains were run for a total of 500,000 iterations and the first 100,000 iterations were discarded and the remaining iterations were thinned by a factor of 100 . The final sample size for each chain comprised 4000 values. MCMC posterior distributions were visually inspected for multiple peaks and Gelman-Rubin test statistics were calculated to ensure chain convergence. Multiple peaks in the posterior distribution or Gelman-Rubin test statistics $>1.1$ were subject to nonconvergence and chains were run for additional iterations to try to achieve convergence.

Model performance was evaluated by comparing the 
median parameter estimates produced for the first year of each scenario to the known parameters used to simulate the data. Estimated median values and credible interval characteristics of posterior parameter distributions were examined to assess strata-specific and total yearly abundance estimates. Total yearly abundance estimates and corresponding credible intervals were calculated by randomly sampling one value from the posterior abundance distribution each unique stratum for the first year of the scenario. The number of unique values is dependent on how many strata are in the year-35 for this simulation. Summing these values and reiterating the random sampling procedure 50,000 times creates a total yearly abundance distribution, $\hat{U}_{\text {Tot }}$. Yearly model bias was measured by the difference of $U_{\text {Tot }}$ from the known $U_{\text {Tot }}$. Strata-specific accuracy was judged on the number of strata that included the known abundance parameter within the predicted $95 \%$ credible intervals. For abundance estimates produced for the 2 real data sets, point estimates and credible interval widths were used to evaluate relative performance among the models. To imitate a naive LincolnPeterson estimator, the $\mathrm{M}_{\mathrm{PS}}$ posterior parameter distributions had portions removed that corresponded to strata missing data.

\section{Results}

\section{Simulation and scenarios}

Markov chains converged for all models and produced representative posterior distributions for parameters with the exception of the $\mathrm{M}_{\mathrm{PS}}$ model. The $\mathrm{M}_{\mathrm{PS}}$ model had Gelman-Rubin test statistics $>1.1$ and density plots with multiple peaks for posterior distributions when strata were missing data. The $\mathrm{M}_{\mathrm{PS}}$ model relied primarily on the vague prior $U$ parameter distributions to construct posterior distributions when data were missing and MCMC required additional iterations $(100,000)$ to converge around the highest density sample space and achieve Gelman-Rubin test statistics $<1.1$. The posterior $U$ distributions obtained from missing strata by using the $\mathrm{M}_{\mathrm{PS}}$ model were largely the product of the prior $U$ distribution and added little relevant biological information to the study, and therefore these strata were removed from the analysis. This exclusion of strata is also illustrative of typical Lincoln-Petersen model performance in that strata without data are excluded from total abundance estimates even if fish are known to be migrating.

The pooled probability model, $\mathrm{M}_{\mathrm{PS}}$, produced the most precise yearly abundance estimates from the simulated scenarios with credible interval widths between $5-9 \%$ of the $\hat{U}_{\text {Tot }}$ (Table 1). The $\mathrm{M}_{\mathrm{PS}}$ precision is misleading in that the uncertainty associated with the stratum missing data was excluded from the total yearly abundance estimate. In addition, the precision of the MPS model is dependent on the assumption that capture probabilities are constant across all strata, which was not true. The inflated precision of the $\mathrm{M}_{\mathrm{PS}}$ model also caused known parameters to be excluded from strata-specific $95 \%$ credible intervals and to result in $\mathrm{M}_{\mathrm{PS}}$ having the worst strata-specific coverage. The $\mathrm{M}_{\mathrm{PS}}$ model overestimated $\hat{U}_{\text {Tot }}$ by 2467 individuals $(10.4 \%)$ for the full scenario. In subsequent scenarios, total yearly abundance estimates became less biased as strata were removed and data were reduced. By removing strata missing data, the $\mathrm{M}_{\mathrm{PS}}$ model $\hat{U}_{\mathrm{Tot}}$ should theoretically become negatively biased by 2093 individuals $(8.9 \%)$ when missing 4 strata in the spring and by 1200 individuals $(5.1 \%)$ when missing 8 strata in the summer. In these scenarios, the nature of the pooled capture probabilities overestimating $\hat{U}_{\text {Tot }}$ offset the negative bias incurred from removing strata with missing data.

The $\mathrm{M}_{\mathrm{HW}}$ and $\mathrm{M}_{\text {SPLINE }}$ models performed better than $\mathrm{M}_{\mathrm{PS}}$ when addressing sparse and missing data (Table 1). The $\mathrm{M}_{\mathrm{HW}}$ model had a credible interval width of $13 \%$ for the full data scenario with a bias of 543 individuals $(2.3 \%)$. As data were reduced and removed, bias increased up to 7064 individuals $(30.0 \%)$ and the percent credible interval width increased up to $63 \%$. The hierarchical structure of the $\mathrm{M}_{\mathrm{HW}}$ model integrated information from the entirety of the year, causing additional variability to be incorporated into the posterior distributions, particularly for strata missing data. Similar to the $\mathrm{M}_{\mathrm{HW}}$ model, the $\mathrm{M}_{\text {SPLINE }}$ model used information from throughout the year to inform strata with sparse and missing data but implemented a P-spline function to localize interpolation of abundance estimates to adjacent strata. This process reduced the variability of posterior parameter distributions for strata with sparse and missing data and produced abundance estimates that were biased from -818 individuals $(-3.5 \%)$ to 516 individuals $(2.2 \%)$ with credible interval widths that were $18-23 \%$ that of $\hat{U}_{\text {Tot }}$. The predetermined spline characteristics prevented the $\mathrm{M}_{\text {SPLINE }}$ model from producing estimates for periods missing $>4$ consecutive strata. The $\mathrm{M}_{\mathrm{HW}}$ and $\mathrm{M}_{\text {SPLINE }}$ model had comparable numbers of strata-specific 95\% credible interval bounds that included known abundance parameters but the credible interval bounds with the $\mathrm{M}_{\text {SPLINE }}$ model were more precise.

The $\mathrm{M}_{\mathrm{HB}}$ model had the most accurate $U$ Tot estimates in 3 scenarios and the second smallest credible interval widths (Table 1). Strata-specific credible intervals produced by the $\mathrm{M}_{\mathrm{HB}}$ model where the only credible intervals to encompass the known parameters for each strata in every scenario. As the quality of simulated data sets decreased, the hierarchical multiyear structure was able to draw inferences from previous years to supplement the missing and sparse data. This procedure allowed model $\mathrm{M}_{\mathrm{HB}}$ to produce the most accurate estimates with missing data.

\section{Application of models to Marsh Creek and Big Creek data}

Total population estimates for Marsh Creek during 2014 were similar among the models, although confi- 


\section{Table 1}

For the simulated data scenario produced by 4 competing models, total yearly abundance estimates $\left(\hat{U}_{\text {Tot }}\right)$ for juvenile Chinook salmon (Oncorhynchus tshawytscha) in Idaho, bias from true $U_{\text {Tot }}(23,527$ individuals), 95\% credible intervals (CIs), credible interval width in relation to the total yearly abundance estimate as a percentage (\% CI width), and the percentages of temporal strata with 95\% CI bounds that covered the true value of unmarked abundance (Strata coverage \%). The $\mathrm{M}_{\mathrm{PS}}$ model structure includes pooled capture probability and simple abundance parameters, the $\mathrm{M}_{\mathrm{HW}}$ model has a hierarchical within-year parameter structure, and the $\mathrm{M}_{\text {SPLINE }}$ model uses a P-spline hierarchical prior for unmarked abundance. The hierarchical multiyear $\mathrm{M}_{\mathrm{HB}}$ model allows information about capture probability and abundance parameters during the same temporal period to be shared among years. Data scenarios may have excluded data for 4 or 8 temporal strata and may have reduced the number of marked and recaptured individuals by $60 \%$ across all strata. Because of predetermined P-spline knot characteristics, the $\mathrm{M}_{\mathrm{SPLINE}}$ model was not able to produce estimates for data missing 8 strata.

\begin{tabular}{|c|c|c|c|c|c|c|}
\hline Models & Data scenario & $\hat{U}_{\text {Tot }}$ & Bias & $95 \% \mathrm{CI}$ & $\begin{array}{l}\% \mathrm{CI} \\
\text { width }\end{array}$ & $\begin{array}{c}\% \text { coverage } \\
\text { of strata }\end{array}$ \\
\hline \multirow[t]{4}{*}{$\mathrm{M}_{\mathrm{PS}}$} & Full & 25,944 & 2467 & $(25,214,26,685)$ & 6 & 34.3 \\
\hline & 4 strata $^{1}$ & 23,059 & -418 & $(22,455,23,682)$ & 5 & 77.1 \\
\hline & 4 strata $60 \%$ reduction ${ }^{\dagger}$ & 24,205 & 728 & $(23,351,25,163)$ & 7 & 85.7 \\
\hline & 8 strata $60 \%$ reduction ${ }^{\dagger}$ & 26,640 & 3163 & $(25,528,27,841)$ & 9 & 31.4 \\
\hline \multirow[t]{4}{*}{$\mathrm{M}_{\mathrm{HW}}$} & Full & 24,020 & 543 & $(22,484,25,717)$ & 13 & 0.8 \\
\hline & 4 strata & 24,492 & 1015 & $(22,412,35,035)$ & 52 & 88.6 \\
\hline & 4 strata $60 \%$ reduction & 23,934 & 457 & $(21,771,34,895)$ & 55 & 88.6 \\
\hline & 8 strata $60 \%$ reduction & 30,541 & 7064 & $(25,780,45,167)$ & 63 & 82.9 \\
\hline \multirow[t]{4}{*}{$\mathrm{M}_{\text {SPLINE }}$} & Full & 23,993 & 516 & $(21,939,26,298)$ & 18 & 82.9 \\
\hline & 4 strata & 22,659 & -818 & $(20,955,24,586)$ & 16 & 77.1 \\
\hline & 4 strata $60 \%$ reduction & 22,697 & -780 & $(20,401,25,606)$ & 23 & 77.1 \\
\hline & 8 strata $60 \%$ reduction & - & - & - & - & - \\
\hline \multirow[t]{4}{*}{$\mathrm{M}_{\mathrm{HB}}$} & Full & 23,245 & -232 & $(22,538,23,976)$ & 6 & 100 \\
\hline & 4 strata & 23,216 & -261 & $(22,517,23,968)$ & 6 & 100 \\
\hline & 4 strata $60 \%$ reduction & 22,509 & -968 & $(21,536,23,594)$ & 9 & 100 \\
\hline & 8 strata $60 \%$ reduction & 22,552 & -925 & $(21,534,23,698)$ & 10 & 100 \\
\hline
\end{tabular}

${ }^{1}$ Posterior parameter distributions for strata that were missing data were removed from the analysis to simulate a naive Lincoln-Peterson estimator.

\section{Table 2}

Total abundance estimate $\left(\hat{U}_{\text {Tot }}\right), 95 \%$ credible intervals (CIs), absolute CI width, and percent CI width for rotary screw trap mark-recapture data collected for juvenile Chinook salmon (Oncorhynchus tshawytscha) at Marsh Creek and Big Creek, Idaho, in 2014 from the pooled-simple $\left(\mathrm{M}_{\mathrm{PS}}\right)$, hierarchical within-year $\left(\mathrm{M}_{\mathrm{HW}}\right)$, hierarchical penalized-spline $\left(\mathrm{M}_{\mathrm{SPLINE}}\right)$, and hierarchical multiyear $\left(\mathrm{M}_{\mathrm{HB}}\right)$ models.

\begin{tabular}{|c|c|c|c|c|}
\hline Model & $\hat{U}_{\text {Tot }}$ & $95 \% \mathrm{CI}$ & CI width & $\%$ CI width \\
\hline \multicolumn{5}{|l|}{ Marsh Creek } \\
\hline $\mathrm{M}_{\mathrm{PS}}{ }^{1}$ & 104,594 & $(103,149,106,064)$ & 2915 & 3 \\
\hline $\mathrm{M}_{\mathrm{HW}}$ & 121,718 & $(110,295,208,526)$ & 98,231 & 81 \\
\hline $\mathrm{M}_{\text {SPLINE }}$ & 112,299 & $(105,910,120,212)$ & 14,302 & 13 \\
\hline $\mathrm{M}_{\mathrm{HB}}$ & 114,035 & $(106,991,123,920)$ & 16,929 & 15 \\
\hline \multicolumn{5}{|l|}{ Big Creek } \\
\hline $\mathrm{M}_{\mathrm{PS}}^{\dagger}$ & 106,141 & $(101,856,104,729)$ & 2873 & 3 \\
\hline $\mathrm{M}_{\mathrm{HW}}$ & 216,292 & $(154,931,584,029)$ & 425,498 & 197 \\
\hline $\mathrm{M}_{\text {SPLINE }}$ & NA & NA & NA & NA \\
\hline $\mathrm{M}_{\mathrm{HB}}$ & 148,110 & $(119,758,239,889)$ & 120,131 & 81 \\
\hline
\end{tabular}

${ }^{1}$ Posterior parameter distributions for temporal strata that were missing data were removed from the analysis. 


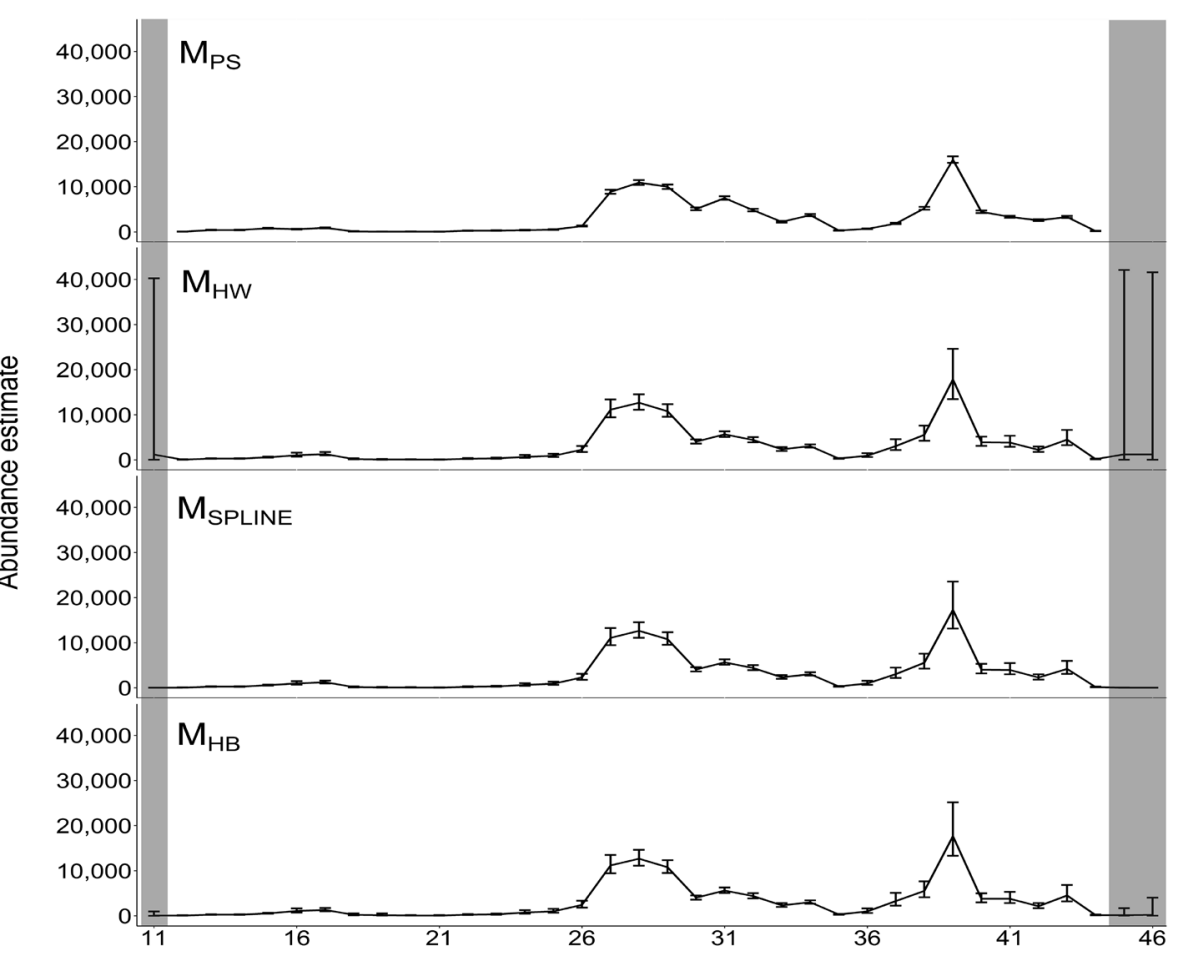

Figure 1

Abundance of juvenile Chinook salmon (Oncorhynchus tshawytscha) emigrating downstream during 2014 by ordinal week, estimated by using 4 competing models with data collected at a rotary screw trap deployed in Marsh Creek, Idaho, in 2014. The 4 models are the pooled-simple $\left(\mathrm{M}_{\mathrm{PS}}\right)$, hierarchical within-year $\left(\mathrm{M}_{\mathrm{HW}}\right)$, hierarchical penalized-spline ( $\left.\mathrm{M}_{\text {SPLINE }}\right)$, and hierarchical multiyear $\left(\mathrm{M}_{\mathrm{HB}}\right)$ models. Gray regions denote estimates produced for temporal strata with missing data in 2014.

dence intervals did not overlap for all (Table 2). The $\mathrm{M}_{\mathrm{PS}}$ model estimated total population abundance at levels 8000-17,000 fish less than the 3 other models but had the smallest $95 \%$ credible interval width. The total population abundance estimate for $\mathrm{M}_{\mathrm{PS}}$ excluded potential fish migrating in ordinal weeks 11,45 , and 46 (Fig. 1). In addition, the precision of $\mathrm{M}_{\mathrm{PS}}$ model estimates rely on the assumption that capture probabilities are constant across all weeks throughout the year and this condition is not likely satisfied because of fluctuating environmental and biological conditions. The $\mathrm{M}_{\mathrm{HW}}$ model had the largest credible interval width that was nearly as large as the total population abundance estimate. Most of the uncertainty around the total population abundance estimate was acquired from strata with missing data at the beginning and end of the year. Posterior distributions for strata missing data using the $\mathbf{M}_{\mathrm{HW}}$ model relied on capture probabilities and abundance characteristics from strata across the entire sample season. Models $\mathbf{M}_{\mathrm{HB}}$ and $\mathbf{M}_{\text {SPLINE }}$ had similar total population estimates but the $\mathrm{M}_{\mathrm{HB}}$ model produced $95 \%$ credible intervals wider by roughly 2500 individuals.
In contrast to estimates for Marsh Creek, total population estimates for Big Creek for 2014 varied greatly among models (Table 2). The $\mathrm{M}_{\mathrm{PS}}$ model estimated total population abundances using 28 out of the 37 strata owing to the removal of strata missing data (Fig. 2). As with results for Marsh Creek, the precision associated with the total population estimate for the $M_{P S}$ model is dependent on the assumption of homogeneous capture probabilities throughout the year and is likely overstated in this application. The population estimate produced by the MHW model was 216,291 fish with a credible interval width nearly double the median estimate. The variability of abundance estimates and capture probabilities throughout the year at Big Creek increased the uncertainty associated with estimates for missing data from the $\mathrm{M}_{\mathrm{HW}}$ model. The $\mathrm{M}_{\text {SPLINE }}$ model was not able to run because of the large number of consecutive strata missing data. The $\mathrm{M}_{\mathrm{HB}}$ model produced a $\hat{U}_{\text {Tot }}$ of 148,110 individuals with a $95 \%$ credible interval width of 120,131 fish.

To illustrate how the $\mathrm{M}_{\mathrm{HB}}$ model used past data to inform the 2014 estimates at the Big Creek RST during the missing strata, we show $u, m$, and $n$ from the 


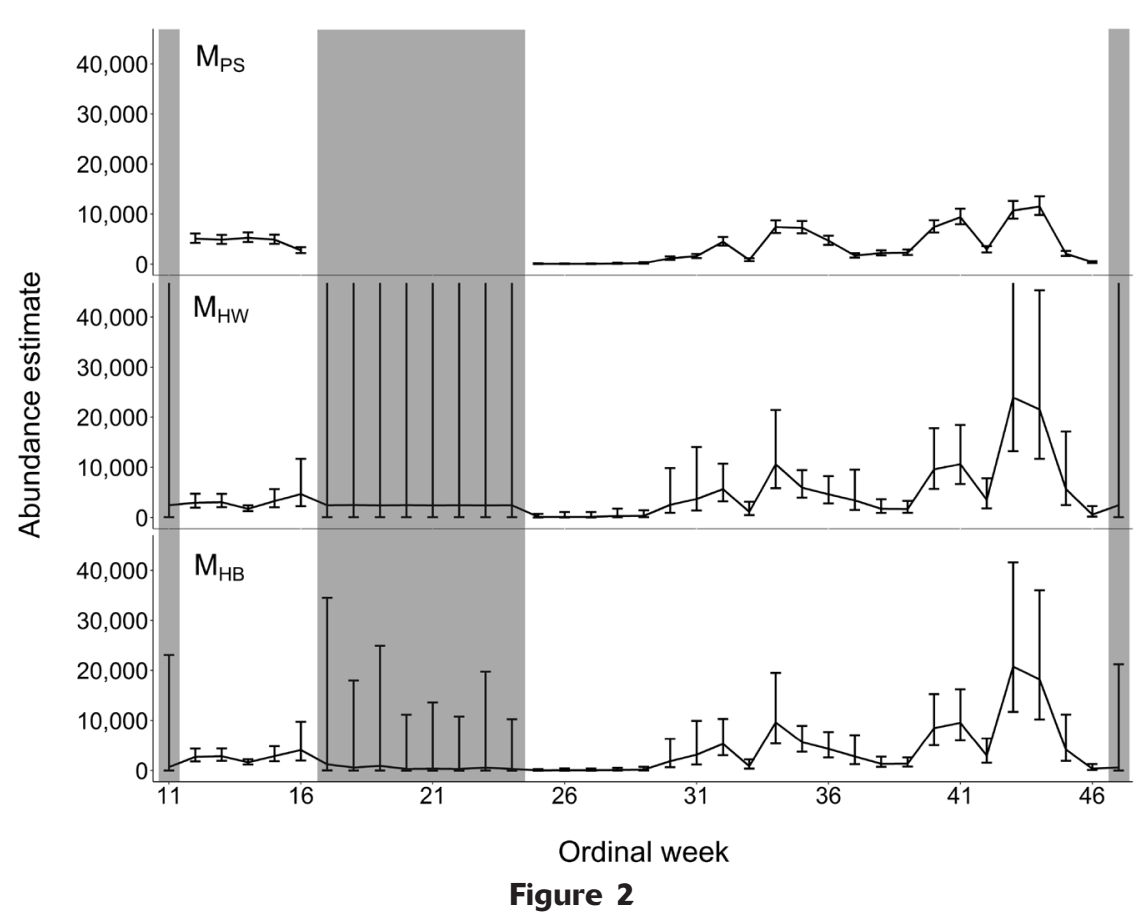

Abundance of juvenile Chinook salmon (Oncorhynchus tshawytscha) emigrating downstream during 2014 by ordinal week, estimated by using 3 competing models with data collected at a rotary screw trap for Big Creek, Idaho, in 2014. The 3 models are the pooled-simple $\left(\mathrm{M}_{\mathrm{PS}}\right)$, hierarchical within-year $\left(\mathrm{M}_{\mathrm{HW}}\right)$, and hierarchical multiyear $\left(\mathrm{M}_{\mathrm{HB}}\right)$ models. A fourth model, the hierarchical penalized-spline $\mathrm{M}_{\text {SPLINE }}$ model, was unable to run and is not displayed. Gray regions denote estimates produced for temporal strata with missing data in 2014 .

past record during the spring (Fig. 3). Spring peaks in catch varied in both timing and magnitude. Capture probabilities were variable but usually $<0.10$. Most gaps in operations start on the descending limb of the catch. These features influenced the 2014 estimates for weeks 17-19 such that the variable peak catches from the past record cause broad $95 \%$ credible intervals (Fig. 2). There were less past data after those strata but catches were not as variable; hence $95 \%$ credible intervals for strata 20-24 are not as wide. Note, however, that there were some data in every stratum in the past record and the $\mathrm{M}_{\mathrm{HB}}$ model was able to use that information to estimate abundance with a precision measure during strata 17-24 in 2014. Because the past record is thin during these periods, the $\mathrm{M}_{\mathrm{HB}}$ model estimate incorporates that uncertainty in the form of wide credible intervals.

\section{Discussion}

To our knowledge, this is the first time a hierarchical Bayesian model has been structured to incorporate data on annually recurring species behavior by using multiple years of data to improve abundance estimates from sparse and missing mark-recapture data. In the simulation, the hierarchical multiyear model produced comparable estimates to those of the best model when data were complete but also produced the most accurate and precise estimates when large periods of data were missing or reduced. Because the hierarchical multiyear model can incorporate information about recurring species behavior to improve the accuracy of abundance estimation, it can be applied beyond salmonids. Studies focused on species that express recurring behavior, particularly for species with low abundances, could benefit from similar hierarchical multiyear models to increase the accuracy and precision of abundance estimates obtained from sparse and missing mark-recapture data.

Current Lincoln-Petersen estimator approaches used for juvenile salmonids abundance estimation do not provide an effective method for addressing sparse or missing data. When strata have low numbers of recaptured individuals (less than 7), confidence intervals obtained from bootstrap approaches are largely uninformative (Steinhorst et al., 2004). Manual pooling, or the use of software that attempts to optimize the balance between satisfying model assumptions while maintaining fine-resolution run characteristics (e.g., Darroch analysis with rank reduction; Bjorkstedt ${ }^{4}$ ), of-

\footnotetext{
${ }^{4}$ Bjorkstedt, E. P. 2000. DARR (Darroch analysis with rank-reduction): a method for analysis of stratified mark-
} 


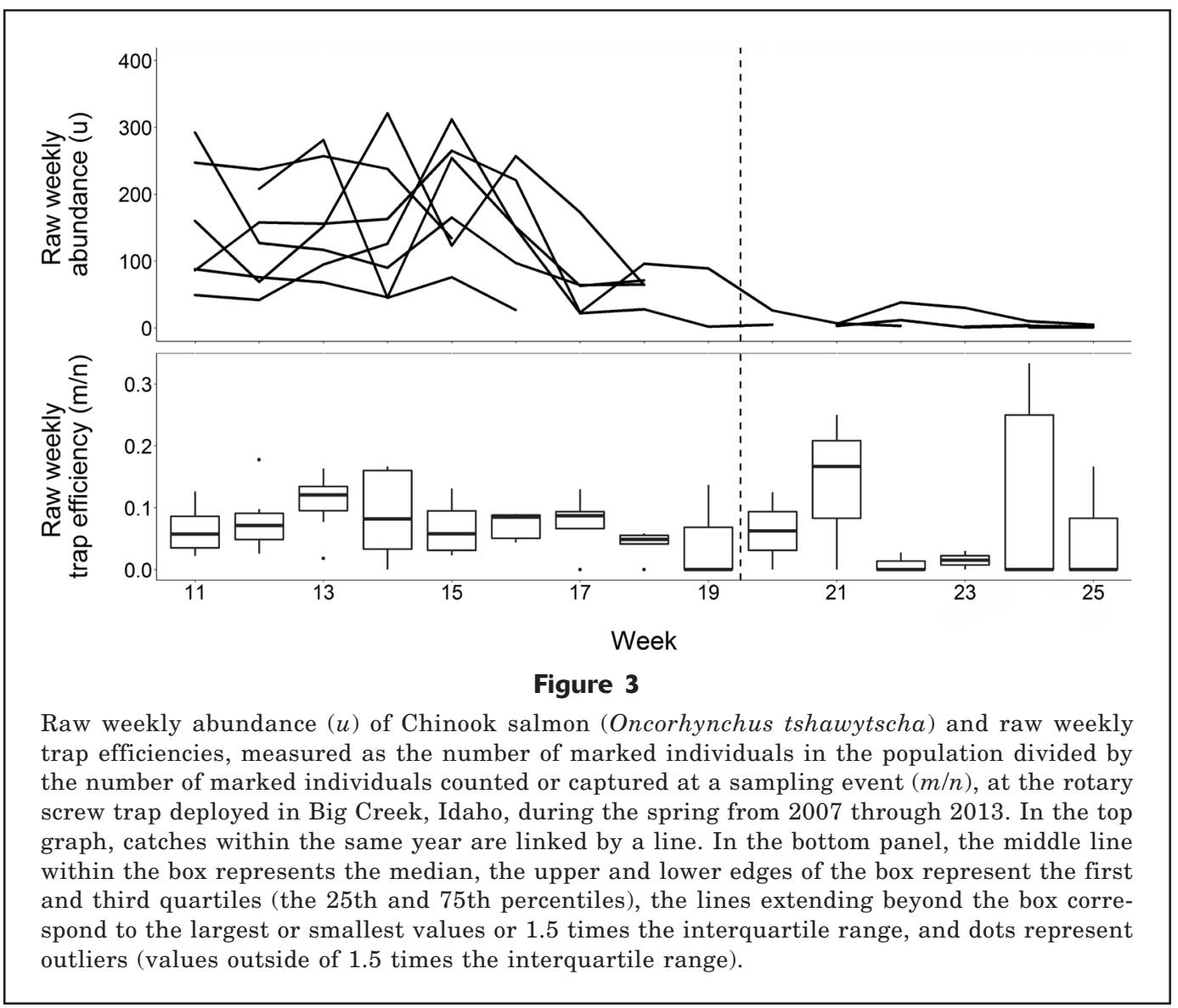

ten results in completely pooled strata, and therefore loses the power to distinguish real changes in sampling efficiency. Additionally, neither approach addresses missing periods when the trap is not able to operate but fish are known to be migrating, as was the case with the $\mathrm{M}_{\mathrm{PS}}$ model in our study that was designed to represent these models. In the simulation, some of the $\mathrm{M}_{\mathrm{PS}}$ model bias was offset by removal of missing strata. Because the $\mathrm{M}_{\mathrm{PS}}$ model can use data only from sampled strata within a single year, it will contain inherent bias. Therefore the $\mathrm{M}_{\mathrm{PS}}$ model precisions and biases reported in Tables 1 and 2 are misleading, because the model structure cannot incorporate the uncertainty associated with the missing strata. When data are not abundant and "well behaved," more complex models are necessary.

The hierarchical structures of the $\mathrm{M}_{\mathrm{SPLINE}}$ and $\mathrm{M}_{\mathrm{HB}}$ models incorporate information about the characteristics of salmonid migration to improve abundance estimates in situations with sparse or missing data. The $\mathrm{M}_{\mathrm{HW}}$ and $\mathrm{M}_{\text {SPLINE }}$ models share information among temporal strata within years. The $\mathrm{M}_{\mathrm{HB}}$ model shares

recapture data from small populations, with application to estimating abundance of smolts from outmigrant trap data. NOAA, Natl. Mar. Fish. Serv., Southwest Fish. Sci. Cent. Admin. Rep. SC-00-02, 19 p. [Available from website.] information during the same temporal period between years, allowing data from previous years to inform periods of sparse or missing data. Because the $\mathrm{M}_{\mathrm{HB}}$ model shares information among years, it effectively uses the entire data set.

Performance during the spring 2014 period in Big Creek shows best how the $\mathrm{M}_{\mathrm{HB}}$ model is useful. The basic Lincoln-Petersen approach as implemented in the $\mathrm{M}_{\mathrm{PS}}$ model provided no estimates in missing strata. Information from within 2014 was not very useful with the 2-month gap in operations, such that the $\mathrm{M}_{\mathrm{HW}}$ model had extremely wide credible intervals and spline components of the $\mathrm{M}_{\text {SPLINE }}$ model could not bridge the missing period. The $\mathrm{M}_{\mathrm{HB}}$ model provided an estimate based on data from previous years and included the appropriate uncertainty around that estimate (e.g., higher variability between years leads to increased uncertainty for estimates with missing data during that time period). The spring peak in migration becomes more pronounced closer to the coast and at lower elevations (Spence and Hall, 2010). hence the problems we address here may be greater elsewhere. Models that performed poorly with our data sets would fare even worse in more extreme environmental scenarios.

For the applications made in this study, we relied only on mark-recapture data but one could incorporate other types of information to make further improve- 
ments. For example, spawner abundance, flow characteristics, and data from co-migrating species could help explain variability in $U$ and $p$ in the respective examples. In particular, data on the number of previous-year spawners could explain annual differences in juvenile abundances, thus accounting for significant changes in abundances and timing of migration from year to year. Inclusion of data from co-migrating species could bolster estimates for species with extremely low abundances. With appropriate assumptions, a multi-species model could be a powerful approach to obtain information for threatened and endangered species.

The framework of the $\mathrm{M}_{\mathrm{HB}}$ model is flexible and can easily be elaborated upon in other ways. For another example, one could combine the $\mathbf{M}_{\text {SPLINE }}$ model and $\mathrm{M}_{\mathrm{HB}}$ model to create a multiyear spline model to apply within- and between-year information. Strata with consistent capture probabilities between years that would potentially benefit the most from the multiyear spline model may not improve much because capture and recapture rates are typically high and data are abundant in these strata. In addition, the use of the multiyear spline model would still be limited by large periods of missing data and running such a model would take substantial computing power and time. We surmise that it would be more beneficial to include environmental and biological covariates into the $\mathrm{M}_{\mathrm{HB}}$ model than creating a multiyear spline model. We chose not to investigate the hybrid spline model or to include covariates because we wanted to illustrate the basic concept and functionality of the competing models and because additional data are not always available. However, if conditions are extreme, then these more complex options may be desirable.

Throughout this study we focused on 2 long-term monitoring projects with data sets ranging from 8 to 21 years; the $\mathrm{M}_{\mathrm{HB}}$ model could produce estimates for missing data with fewer years of data. The accuracy and precision of abundance estimates produced for periods with sparse or missing data from short data sets will depend on the amount of data collected during years that the trap was able to operate and the consistency of recurring life-history characteristics between years. The effects of relevant factors on the quality of information in the data record can be tested but that is beyond the scope of this article. However, so long as the assumption that the species exhibits a recurring life-history characteristic that is expressed during the same temporal period between years, the estimates from sparse and missing data would be accurate but the uncertainty with these estimates will be expressed in wide, credible interval widths.

The hierarchical multiyear Bayesian model has broad application to fish and wildlife studies that employ mark-recapture approaches to obtain population abundance estimates, particularly when addressing issues of sparse or missing data. Other studies have used similar Bayesian approaches to calculate adult salmonid abundance estimates in Alaska (Sethi and Tanner, 2013), survival estimates of harbor seals (Phoca vitu- lina) in Scotland (Mackey et al., 2008), and wolverine (Gulo gulo) densities in Alaska (Royle et al., 2011) in order to address issues associated with sparse data. In this study, we were able to produce abundance estimates for populations of anadromous salmonids with sparse data by structuring a hierarchical model that incorporated prior biological information about the species behavior. The $\mathrm{M}_{\mathrm{HB}}$ model has applicability to a wide range of fish and wildlife research that uses mark-recapture data to estimate species abundance. This model will be particularly useful in assessments of species for which long-term monitoring has occurred but for which low abundances and variable environmental conditions affect sampling efforts.

\section{Acknowledgement}

We thank the Idaho Department of Fish and Game (IDFG), University of Idaho, and Bonneville Power Administration (projects 1989-098-00, 1990-055-00 and 1991-073-00) for the opportunity and financial support to conduct this research. We greatly appreciate the discussion, reviews, and feedback provided by M. Quist, R. Kinzer, members of B. Kennedy's laboratory, and numerous other fisheries biologists from the IDFG and Nez Perce tribe. We are particularly grateful to K. Apperson, L. Jansen, D. Venditti, and B. Barnett for providing the RST data that made the analysis possible.

\section{Literature cited}

Bjornn, T. C.

1971. Trout and salmon movements in two Idaho streams as related to temperature, food, stream flow, cover, and population density. Trans. Am. Fish. Soc. 100:423-438. Article

1978. Survival, production, and yield of trout and Chinook salmon in the Lemhi River, Idaho. Univ. Idaho, Coll. For., Wildl. Range Sci. Bull. 27, 57 p.

Bonner, S. J., and C. J Schwarz.

2011. Smoothing population size estimates for timestratified mark-recapture experiments using Bayesian P splines. Biometrics 67:1498-1507. Article

Calambokidis, J., and J. Barlow.

2004. Abundance of blue and humpback whales in the eastern North Pacific estimated by capture recapture and line-transect methods. Mar. Mamm. Sci. 20:63-85. Article

Copeland, T. S., D. A. Venditti, and B. R. Barnett.

2014. The importance of juvenile migration tactics to adult recruitment in stream-type Chinook salmon populations. Trans. Am. Fish. Soc. 143:1460-1475. Article

Ellison, A. M.

2004. Bayesian inference in ecology. Ecol. Letters, 7:509-520. Article

Fryxell, J. M., A. R. E. Sinclair, and G. Caughley.

2014. Wildlife ecology, conservation, and management, $3^{\text {rd }}$ ed., 509 p. John Wiley \& Sons Ltd., Oxford, UK.

Johnson, D. H., B. M. Shrier, J. S. O’Neal, J. A. Knutzen, X. Augerot, T. A. O’Neil, T., and T. N. Pearsons.

2007. Salmonid field protocols handbook: techniques for 
assessing status and trends in salmon and trout populations, 478 p. Am. Fish. Soc., Bethesda, MD.

Kareiva, P. M.

1983. Local movement in herbivorous insects: applying a passive diffusion model to mark-recapture field experiments. Oecologia. 57:322-327. Article

Lang, S., and A. Brezger.

2004. Bayesian P-splines. J. Comput. Graph. Stat. 13:183212. Article

Mackey, B. L., J. W. Durban, S. J. Middlemas, and P. M. Thompson.

2008. A Bayesian estimate of harbour seal survival using sparse photo-identification data. J. Zool. 274:18-27. Article

Mäntyniemi, S., and A. Romakkaniemi.

2002. Bayesian mark-recapture estimation with an application to a salmonid smolt population. Can. J. Fish. Aquat. Sci. 59:1748-1758. Article

Mill, L. S.

2007. Conservation of wildlife populations: demography, genetics, and management, 407 p. Blackwell Publishing, Malden, MA.

Mowat, G., and C Strobeck.

2000. Estimating population size of grizzly bears using hair capture, DNA profiling, and mark-recapture analysis. J. Wildl. Manage. 64(1):183-193. Article

Nichols, J. D.

1992. Capture-recapture models. BioScience 42:94-102. Article

Otis, D. L., K. P. Burnham, G. C. White, and D. R. Anderson. 1978. Statistical inference from capture data on closed animal populations. Wildl. Soc., Wildl. Monogr. 62, 135 p.

Plummer, M.

2003. JAGS: a program for analysis of Bayesian graphical models using Gibbs sampling. In Proceedings of the $3^{\text {rd }}$ international workshop on distributed statistical computing (DSC 2003); Vienna, Austria, 20-22 March (K. Hornik, F. Leisch, and A Zeileis, eds.), 10 p. [Available from website.]

$\mathrm{R}$ Core Team.

2015. R: a language and environment for statistical com- puting. R Foundation for Statistical Computing, Vienna, Austria. [Available from website, accessed September 2015].

Royle, J. A., and R. M. Dorazio.

2008. Hierarchical modeling and inference in ecology: the analysis of data from populations, metapopulations and communities. Academic Press, Inc., London.

Royle, J. A., A. J. Magoun, B. Gardner, P. Valkenburg, and R. E. Lowell

2011. Density estimation in a wolverine population using spatial capture-recapture models. J. Wildl. Manage. 75:604-611. Article

Schwarz, C. J., and C. G. Taylor.

1998. Use of the stratified-Petersen estimator in fisheries management: estimating the number of pink salmon (Oncorhynchus gorbuscha) spawners in the Fraser River. Can. J. Fish. Aquat. Sci. 55:281-296. Article

Seber, G. A. F.

2002. The estimation of animal abundance and related parameters, $2^{\text {nd }}$ ed., 654 p. Blackburn Press, Caldwell, NJ.

Sethi, S. A., and T. L. Tanner.

2013. Bayesian implementation of a time stratified Lincoln-Petersen estimator for salmon abundance in the upper Matanuska River, Alaska, USA. Fish. Res. 145:90-99. Article

Spence, B. C., and J. D. Hall.

2010. Spatiotemporal patterns in migration timing of coho salmon (Oncorhynchus kisutch) smolts in North America. Can. J. Fish. Aquat. Sci. 67:1316-1334. Article

Steinhorst, K., Y. Wu, B .Dennis, and P. Kline.

2004. Confidence intervals for fish out-migration estimates using stratified trap efficiency methods. J. Agric. Biol. Environ. Stat. 9:284-299. Article

$\mathrm{Su}$, Y.-S., and M. Yajima.

2015. R2jags: using $\mathrm{R}$ to run 'JAGS'. R package, vers. 0.57. [Available from website.]

Zabel, R. W., T. Wagner, J. L. Congleton, S. G. Smith, and J. G. Williams.

2005. Survival and selection of migrating salmon from capture-recapture models with individual traits. Ecol. Appl. 15:1427-1439. Article 